\title{
Comparative Effectiveness of Plantar-Massage Techniques on Postural Control in Those With Chronic Ankle Instability
}

\author{
Erik A. Wikstrom, PhD, ATC, FACSM*; Kyeongtak Song, MS, ATC*; \\ Ashley Lea, ATC†; Nastassia Brown, ATC
}

*University of North Carolina at Chapel Hill; †Florida State University, Tallahassee; $¥$ Midwestern University, Glendale, $\mathrm{AZ}$

Context: One of the major concerns after an acute lateral ankle sprain is the potential for development of chronic ankle instability (CAI). The existing research has determined that clinician-delivered plantar massage improves postural control in those with CAI. However, the effectiveness of self-administered treatments and the underlying cause of any improvements remain unclear.

Objectives: To determine (1) the effectiveness of a selfadministered plantar-massage treatment in those with $\mathrm{CAl}$ and (2) whether the postural-control improvements were due to the stimulation of the plantar cutaneous receptors.

Design: Crossover study.

Setting: University setting.

Patients or Other Participants: A total of 20 physically active individuals (6 men and 14 women) with self-reported CAI.

Intervention(s): All participants completed 3 test sessions involving 3 treatments: a clinician-delivered manual plantar massage, a patient-delivered self-massage with a ball, and a clinician-delivered sensory brush massage.

Main Outcome Measure(s): Postural control was assessed using single-legged balance with eyes open and the Star Excursion Balance Test.

Results: Static postural control improved $(P \leq .014)$ after each of the interventions. However, no changes in dynamic postural control after any of the interventions were observed $(P$ $>$.05). No differences were observed between a cliniciandelivered manual plantar massage and either a patient-delivered self-massage with a ball or a clinician-delivered sensory brush massage in any postural-control outcome.

Conclusions: In those with CAI, single 5-minute sessions of traditional plantar massage, self-administered massage, and sensory brush massage each resulted in comparable static postural-control improvements. The results also provide empirical evidence suggesting that the mechanism for the posturalcontrol improvements is the stimulation of the plantar cutaneous receptors.

Key Words: self-administered treatment, balance, plantar cutaneous receptors

\section{Key Points}

- Plantar massage improved static postural control in those with chronic ankle instability.

- Self-administered plantar massage was as effective as a clinician-delivered massage in improving static postural control.

- Stimulation of the plantar cutaneous receptors appears to be the reason for the postural-control improvements after plantar massage.

$\mathrm{O}$ ne of the major concerns after an acute lateral ankle sprain is the potential for the development of chronic ankle instability (CAI). This health condition is defined by multiple recurrent sprains and the presence of long-term residual symptoms, ${ }^{1}$ including decreased levels of physical activity. ${ }^{2}$ Researchers ${ }^{3}$ have indicated that up to $74 \%$ of those who sustain an acute lateral ankle sprain develop CAI, a leading cause of posttraumatic ankle osteoarthritis. ${ }^{4}$ Although the exact mechanism of CAI is still unclear, altered sensory input of the ankle and foot is hypothesized to impair sensorimotor function and ultimately lead to the development of CAI. ${ }^{5,6}$ For example, patients with CAI have demonstrated increased light-touch thresholds on the plantar surface of the foot compared with uninjured healthy controls, ${ }^{7-9}$ and this impairment is associated with postural-control deficits. ${ }^{8}$
Due to the high rate of recurrent ankle sprains and the risk of long-term health consequences such as decreased physical activity and osteoarthritis, identifying appropriate treatments for somatosensory dysfunction in patients with CAI is critical.

Balance training is an effective intervention for improving postural control ${ }^{10}$ and reducing rates of recurrent ankle sprains. ${ }^{11}$ Strength training is another traditional treatment for those with CAI; in those with CAI, it improves not only strength but also joint position sense. ${ }^{12}$ Furthermore, unsupervised or at-home programs can be as effective as supervised rehabilitation programs. ${ }^{13}$ As a result, the authors of a recent systematic review ${ }^{13}$ recommended home rehabilitation programs if patients with sprained ankles cannot pursue supervised rehabilitation. 
Whereas traditional treatments target motor function effectively, recent investigators ${ }^{14,15}$ have also attempted to stimulate sensory receptors in order to improve overall sensorimotor function, which is often quantified as postural control. For example, Ross et al $^{16}$ found that combining stochastic resonance (a subsensory white noise) with a 6week coordination training program resulted in greater postural-control improvements than coordination training alone. Similarly, Hoch and McKeon ${ }^{15}$ demonstrated that stimulating capsular or articular receptors (or both) via anteroposterior talocrural joint mobilizations not only resulted in increased dorsiflexion range of motion but also improved eyes-open postural control. A brief 5-minute plantar-massage intervention that consisted of a combination of effleurage and petrissage also improved postural control in those with CAI. ${ }^{14}$ However, the existing research has indicated only that clinician-delivered sensory-targeted interventions improved postural control in those with CAI without considering the possible effectiveness of self- (ie, patient-) administered treatments. In addition, the previously used plantar-massage treatment was hypothesized to work by stimulating the plantar cutaneous receptors, but the technique would also stimulate the underlying musculotendinous receptors. Without knowing which set of receptors is responsible for the observed improvements, we cannot optimize the intervention.

Therefore, 2 primary aims were pursued in this investigation. The first was to determine the effectiveness of a self-administered plantar-massage treatment relative to a clinician-administered plantar massage known to improve postural control in those with CAI. The second aim was to determine whether the previously observed postural-control improvements were due to the stimulation of the plantar cutaneous receptors or the stimulation of the underlying musculotendinous receptors. We hypothesized that a selfadministered plantar massage would be as effective as a clinician-administered plantar-massage treatment. We also hypothesized that massage-related postural-control improvements would result from stimulating the plantar cutaneous receptors during the intervention.

\section{METHODS}

\section{Design and Participants}

A crossover study with treatment order counterbalanced by a modified Latin square design was used. Participants, administrators, and assessors were not blinded to the treatment received during the investigation. A total of 20 physically active individuals (6 men and 14 women) with self-reported CAI participated in this study (Table 1). Inclusion criteria required participants to be between 18 and 45 years of age, have a history of at least one significant ankle sprain, have sustained at least 2 episodes of "giving way" within the previous 6 months, and score 5 or more yes responses on the Ankle Instability instrument. ${ }^{17}$ The exclusion criteria were failing to meet the inclusion criteria, any known balance or vision problems, having sustained an acute lower extremity or head injury within 6 weeks of testing, any chronic musculoskeletal condition known to affect balance, or a history of musculoskeletal surgeries or fractures to either limb. ${ }^{17}$ Although this investigation was initiated before the recommendations made by the Interna-
Table 1. Participants' Demographics and Injury Characteristics

\begin{tabular}{lr}
\hline Characteristic & Mean \pm SD \\
\hline Age, y & $21.45 \pm 1.76$ \\
Height, cm & $166.49 \pm 8.70$ \\
Weight, kg & $68.53 \pm 14.16$ \\
National Aeronautics and Space Administration & \\
$\quad$ Physical Activity Score & $5.45 \pm 1.96$ \\
No. of yes responses on the Ankle Instability & \\
$\quad$ Instrument & $7.05 \pm 1.23$ \\
No. of total sprains & $5.25 \pm 3.04$ \\
No. of giving-way episodes within the past 6 & \\
$\quad$ months & $4.05 \pm 3.07$ \\
Foot and Ankle Ability Measure score, \% & $89.60 \pm 7.12$ \\
Foot and Ankle Ability Measure-Sport score, \% & $89.10 \pm 6.65$ \\
\hline
\end{tabular}

tional Ankle Consortium, ${ }^{17}$ the aforementioned criteria are in line with those recommendations.

\section{Procedures}

All participants completed 3 test sessions over at least a 3-week period of time. At least a 7-day wash-out period was required between interventions. Each session consisted of a pretest, a treatment, and a posttest, with identical pretesting and posttesting across all sessions. The total amount of time between completion of the pretest assessment and completion of the posttest assessment was roughly 10 minutes. This study was approved by the host university's institutional review board, and at the beginning of the first session, all participants read and signed an informed consent form before we collected general demographic information such as age, height, weight, leg length, and foot size. Participants next completed the Foot and Ankle Ability Measure (FAAM) and the FAAM-Sport (FAAM-S) as well as the National Aeronautics and Space Administration Physical Activity Scale. ${ }^{18,19}$ The FAAM and the FAAM-S measure self-reported function during activities of daily living and sports, respectively, and a higher score represents a higher level of function. Although FAAM and FAAM-S scores were collected, we did not use them as inclusion criteria because we wanted to capture information on those with CAI across a wide range of the self-assessed disability spectrum (FAAM range, 70\%$98.8 \%$; FAAM-S range, 72\%-96.8\%). The National Aeronautics and Space Administration Physical Activity Scale indicates a participant's activity level, and a higher score represents a higher level of physical activity. Participants then completed static and dynamic balance pretest assessments. Static and dynamic assessments were completed in counterbalanced order within each intervention session during both the pretest and posttest.

\section{Static Balance Testing}

Static balance was assessed using an Accusway force plate (Advanced Mechanical Technology Inc, Watertown, MA). The participants performed barefoot, single-legged balance on the force plate with their eyes open for three 10second trials on the involved limb. They were instructed to keep their hands on their hips and their nonstance limb at approximately $30^{\circ}$ of hip flexion and $45^{\circ}$ of knee flexion throughout the trials. The trial was stopped and repeated if there were any faults such as putting the foot down or use of the hands to regain balance. Center-of-pressure (COP) data 


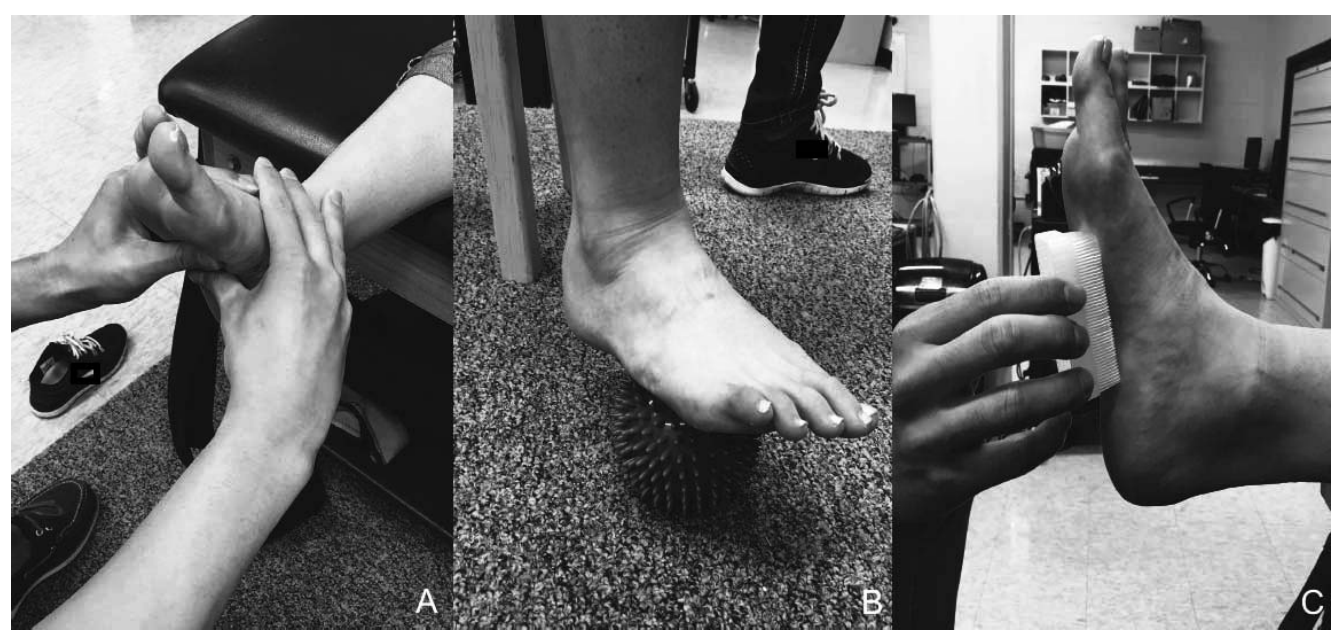

Figure 1. Three treatments were administered: A, manual plantar massage, B, self-administered ball massage, and C, sensory brush plantar massage.

were collected at $50 \mathrm{~Hz}$ using the Balance Clinic Software program provided by the force-plate manufacturer. The static postural-control outcomes were COP velocity (COPV) and COP 95\% area ellipse (COPA-95). The COPV assesses temporal qualities of postural control; a higher score indicates worse postural control. The COPA-95 assesses spatial qualities of postural control, ${ }^{20}$ and higher scores again indicate worse postural control. Average scores for each outcome of 3 successful trials were used for subsequent analysis. Both outcomes have identified group differences between individuals with CAI and uninjured healthy controls. ${ }^{21}$ Intraclass correlation coefficients (ICCs) for COPV during single-legged balance have ranged $^{22}$ from 0.65 to 0.79 . Reliability data, captured from 2 randomly selected pretest assessments, indicated fair to poor reliability for COPV (0.68) and COPA-95 (0.57) over the 3 -week involvement of the participants in the investigation.

\section{Dynamic Balance Testing}

Dynamic balance was measured using the anterior, posteromedial, and posterolateral directions of the Star Excursion Balance Test (SEBT). Participants stood on the affected leg and reached maximally in all 3 directions with the other limb. ${ }^{23}$ The foot was positioned in the middle of the SEBT grid for all 3 directions. Participants repeated trials if they failed to maintain their balance during the trials or if they placed weight on the reach limb. A total of 6 practice trials were completed in each direction before the formal assessment began. ${ }^{23}$ Reach distances were normalized by a participant's leg length (anterior-superior iliac spine to ipsilateral medial malleolus), and the average normalized reach distances for each direction were used for statistical analysis. Previous researchers ${ }^{23}$ demonstrated excellent test-retest reliability for the SEBT $(\mathrm{ICC}=0.84$ 0.92).

\section{Interventions}

After the pretest, 1 of 3 treatments was administered: a clinician-delivered manual plantar massage (MANUAL), a patient-delivered massage using an off-the-shelf massage ball (BALL), or a clinician-delivered massage using a sensory brush (BRUSH; Figure 1). Each treatment lasted 5 minutes. The MANUAL consisted of a combination of pettrisage and effleurage techniques. ${ }^{14}$ Only the plantar surface of the foot was targeted in order to stimulate plantar cutaneous receptors, as reported previously. ${ }^{14}$ No effort was made to standardize either the amount of time dedicated to pettrisage or effleurage or the amount of time that a specific location of the foot was massaged. The BALL required the participant to roll the spiked rubber ball under the entire plantar surface of the foot while seated. No other instructions were given. For the BRUSH, the device was gently stroked over the bottom of the foot while the supine patient lay on a standard treatment table. This technique is designed to stimulate only the plantar cutaneous receptors; thus, extremely light touch was used, and participants were told that the treatment might "tickle." No effort was made to standardize the time spent massaging different parts of the foot. All participants were assigned to 1 of 3 treatment orders (1: MANUAL, BALL, BRUSH; 2: BRUSH, MANUAL, BALL; 3: BALL, BRUSH, MANUAL) in a counterbalanced fashion using the modified Latin square design that were completed over the 3 sessions. As a result, allocation of treatment order was not concealed from the research team, but participants were unaware of the treatment they were to receive until they arrived for their test sessions. After each intervention, participants completed a posttest balance battery that was identical to the pretest methods.

\section{Statistical Analysis}

The independent variables were intervention (MANUAL, BALL, BRUSH) and time (pretest, posttest). The dependent static balance variables were the COPV and COPA-95. Dynamic-balance dependent variables were the normalized reach distances in the anterior, posteromedial, and posterolateral directions of the SEBT. Using SPSS (version 22.0; IBM Corp, Armonk, NY), we calculated 2 separate 2-way, repeated-measures multivariate analyses of variance with a significance level of $P<.05$ to determine the effect of the massage interventions on static and dynamic balance in those with CAI. In the presence of a time effect, post hoc comparisons were conducted using paired $t$ tests. The 
Hedges $g$ effect sizes and $95 \%$ confidence intervals (CIs) for each dependent variable were calculated on the basis of the means, standard deviations, and correlation of the pretest with posttest data for each intervention to demonstrate clinical meaningfulness of the observed changes and confirm differences among the interventions. An effect size of less than 0.3 was interpreted as small, 0.31 to 0.7 as moderate, and greater than 0.71 as large. ${ }^{24}$ Finally, the minimum detectable change (MDC) for each outcome measure was calculated ${ }^{15}$ as the standard error of measurement (standard deviation $\times[1-\mathrm{ICC}]^{1 / 2}$ ) multiplied by $2^{1 / 2}$. The ICC was calculated from 2 of the 3 days' pretest assessments, randomly selected for each outcome, and recorded during the investigation. The $M D C$ illustrates the amount of change needed to be confident that the change is due to the intervention and not random error.

\section{RESULTS}

Group means, standard deviations, and effect sizes of the static and dynamic postural-control outcomes can be seen in Table 2. No significant interactions between intervention and time were detected for static or dynamic postural control $(P>.05)$. A time main effect, indicating an improvement in static postural control, was identified $(P<$ $.001)$. Post hoc testing showed that both COPV and COPA95 improved after each intervention (MANUAL, BALL, and BRUSH; $P \leq .014$ ). Pretest-to-posttest effect sizes indicated clinically meaningful improvements in static postural-control outcomes after the MANUAL, BALL, and BRUSH interventions. The COPV improvements after the BALL and BRUSH interventions exceeded the calculated MDC $(0.46 \mathrm{~cm} / \mathrm{s})$. However, COPV after the MANUAL intervention and COPA-95 $\left(2.62 \mathrm{~cm}^{2}\right)$ after all interventions did not exceed the calculated MDC scores. No time main effect was observed for dynamic postural control $(P>.05)$. Thus, is it not surprising that SEBT scores did not exceed the calculated MDC scores for the anterior $(2.04 \%)$, posteromedial $(2.98 \%)$, and posterolateral $(3.90 \%)$ reach directions.

To address our first aim, we compared the effects of the MANUAL intervention with the effects of the BALL. No differences were present between these interventions in any postural-control outcome because all confidence intervals crossed zero, indicating comparable effectiveness between the interventions (Figure 2A). To address the second aim, we compared the MANUAL intervention with the BRUSH. No differences were seen between the MANUAL and BRUSH interventions in any postural-control outcome because all confidence intervals crossed zero (Figure 2B). This result indicates that stimulating only the plantar cutaneous receptors resulted in similar postural-control improvements relative to a MANUAL intervention that likely stimulated both the plantar cutaneous receptors and underlying muscular receptors.

\section{DISCUSSION}

Our aims were to determine whether a self-administered massage could improve postural control as effectively as a traditional manual massage and whether postural-control improvements from a plantar massage in those with CAI were due to stimulating the cutaneous receptors or the underlying muscular receptors. The main findings of this

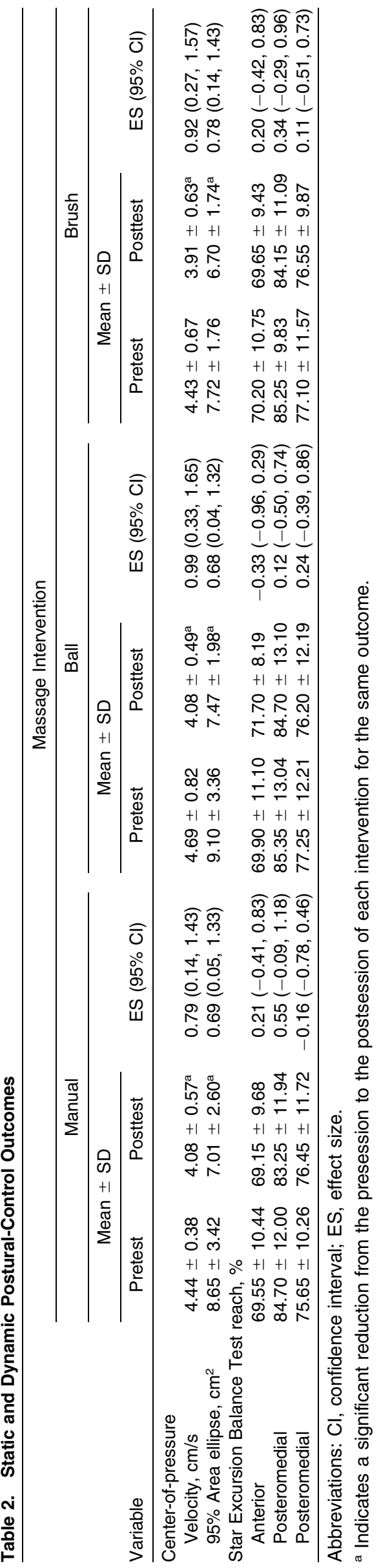



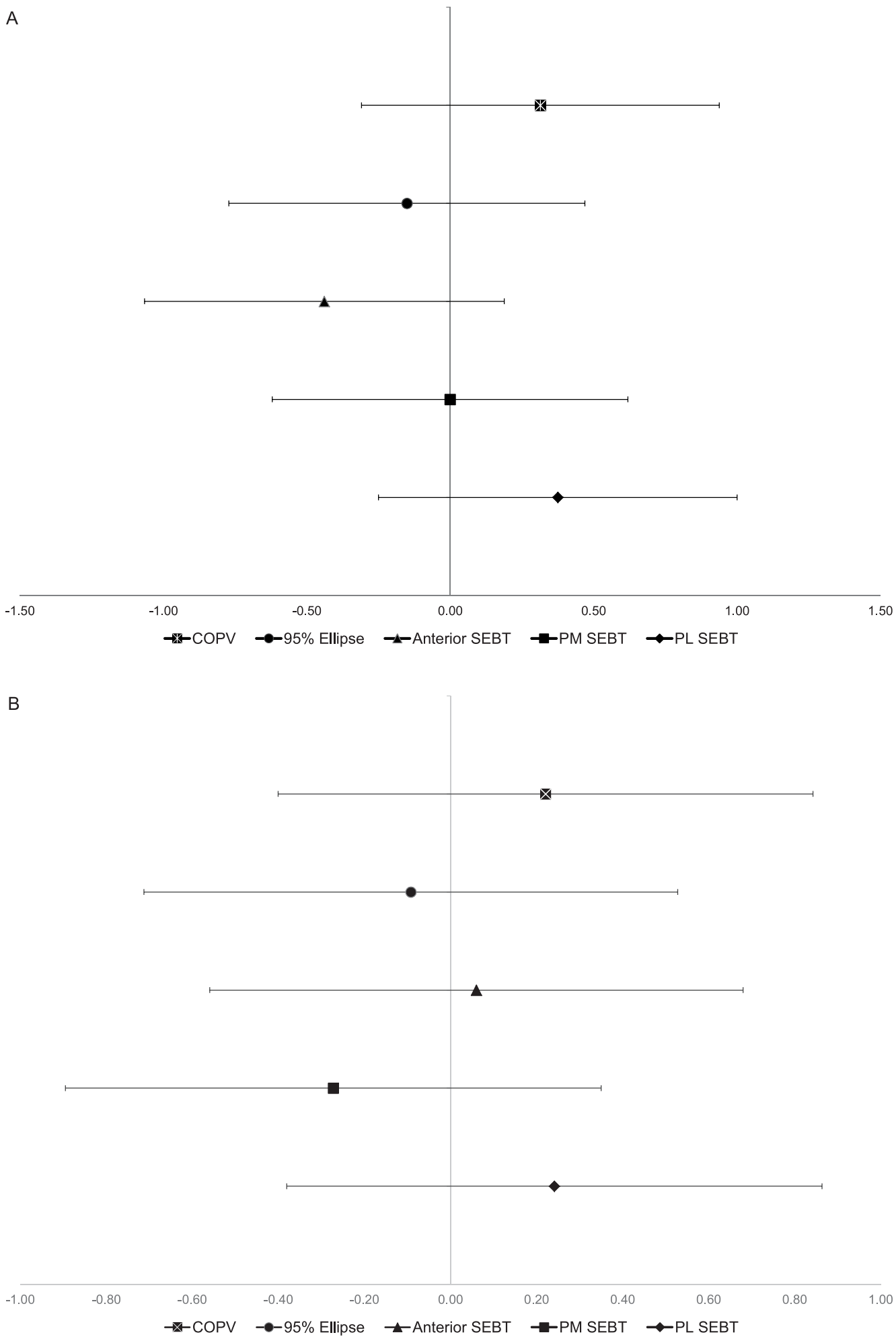

Figure 2. Effect-size comparisons $A$, between the manual massage and the self-administered ball massage and $B$, between the manual massage and the sensory brush. Abbreviations: COPV, center-of-pressure velocity; PL, posterolateral; PM, posteromedial; SEBT, Star Excursion Balance Test.

study were (1) a single bout of plantar massage, regardless of the technique used, improved static postural control, (2) a self-administered massage was as effective as a clinicianadministered manual massage, and (3) stimulation of the plantar cutaneous receptors appeared to be responsible for the improvements in static postural control.
These results are in agreement with those of previous authors $^{14,24}$ who found that a single treatment of a traditional plantar massage improved static postural control in those with CAI. Similarly, six 5-minute traditional massage treatments over 2 weeks resulted in static posturalcontrol and self-assessed functional improvements in those 
with CAI. ${ }^{24}$ Furthermore, the self-assessed functional improvements were maintained for at least 1 month after the massage intervention. ${ }^{24}$ The massage interventions had no effect on dynamic postural control, which is consistent with the work of Hoch and McKeon, ${ }^{15}$ who showed that a single bout of ankle-joint mobilization did not improve performance by those with CAI in any direction of the SEBT. However, 6 joint-mobilization treatments in 2 weeks improved performance by those with CAI in all 3 directions of the SEBT. ${ }^{25}$ Similar results were observed after 3 weeks of joint mobilization in those with CAI, and these improvements were present at the 6-month follow-up. ${ }^{26}$ If these findings are considered cumulatively, we believe they suggest that manual therapies can immediately improve static postural control, perhaps by priming the sensory receptors of the foot-ankle complex as hypothesized by Ross et al. ${ }^{27}$ However, completing more complex motor tasks, such as the SEBT, may require additional treatments to go beyond a simple priming of the sensory system. We speculate that additional treatments may allow for increased or better sensory information (or both) from the foot-ankle complex to be recognized by the central nervous system and integrated with additional sensory and motor information to create more efficient motor programs. ${ }^{28}$ However, further research is needed to confirm this hypothesis.

Whereas traditional plantar massage appears to be an effective intervention for those with CAI, determining the comparable effectiveness of a self-administered massage is important for 2 reasons. First, allied health care providers may not have the time or physical stamina to deliver multiple massage treatments in a clinic session. Second, many people who sustain a lateral ankle sprain do not seek treatment from health care professionals. ${ }^{29}$ Thus, effective, low-cost self-administered treatments could be advantageous. Home and unsupervised rehabilitation programs for those with CAI and acute ankle sprains have been shown to be effective. ${ }^{13}$ The current results demonstrate that a selfadministered plantar massage using an off-the-shelf massage ball is as effective in improving static postural control in those with CAI as a traditional plantar massage. This finding has the potential to positively affect clinical practice in freeing the busy clinician for other treatments or assessments. The results also support a low-cost treatment option for individuals who do not see a health care provider.

Perry et $\mathrm{al}^{30}$ suggested that cutaneous sensation on the plantar surface of the foot plays an important role in dynamic stabilization. Thus, previous researchers ${ }^{14,24}$ have hypothesized that postural-control improvements after plantar massage were due to the stimulation of the plantar cutaneous receptors. However, the traditional plantarmassage treatments used in research likely stimulated the underlying musculotendinous receptors in addition to the plantar cutaneous receptors. We demonstrated comparable postural-control improvements with MANUAL and BRUSH interventions, the latter of which stimulates only the plantar cutaneous receptors. This finding is the first empirical evidence that stimulation of the plantar cutaneous receptors could be the underlying mechanism of posturalcontrol improvements after plantar massage in those with CAI. Similarly, the BRUSH was as effective as the BALL treatment, which is also likely to stimulate the underlying musculature.
It should be noted that we did not study a control or sham group in this investigation, and this is a limitation. In addition, our pretest and posttest assessments were completed in a very short period of time, which introduces the possibility of a learning effect. Finally, not all of our significant findings exceeded the calculated MDC scores, suggesting that the improvements may be due to error. However, we remain confident in our interpretation of the results for 3 reasons. First, previous plantar-massage data gathered using similar pretest-to-posttest time frames (approximately 10 minutes) have consistently shown no static postural-control improvements in a control group. ${ }^{24,31,32}$ This does not eliminate the possibility of a learning effect within our sample but suggests a low probability of such an effect. Second, our pretest-to-posttest effect sizes are comparable with, if not larger than, previously reported between-groups (massage, control) effect sizes. ${ }^{24,31,32}$ Finally, MDC scores are a valuable tool for interpreting the effects of interventions relative to the stability of an outcome. However, the MDC scores used in this investigation were generated from pretest scores that could have been at least 2 weeks apart, compared with the pretest-to-posttest assessments completed within about 10 minutes of each other. Given the fact that both sleep patterns $^{33}$ and time of day can influence postural control, ${ }^{34}$ we are hesitant to refute our findings and large effects on the basis of the calculated MDC scores but believe it is important to include such data in the spirit of full disclosure.

It is important to consider the clinical implications of the current and cumulative Sensory-Targeted Ankle Rehabilitation Strategies (STARS) findings. Cumulatively, the literature demonstrates that multiple STARS treatments have an effect that could last between 1 month and 6 months depending on the exact treatment protocol. $^{24,26}$ How to best use the effects of a single treatment is much less clear because whether the posturalcontrol improvements are retained is unknown. We speculate that the observed improvements would be relatively brief and therefore would not be retained through a practice or other athletic event. But they may be beneficial in certain rehabilitative scenarios. For example, consider a patient who could progress to a more difficult level of a particular motor task during rehabilitation but has demonstrated a great deal of anxiousness and apprehension about his or her ability to recover and return to sport. In this case, a temporary improvement in postural control may be indicated to instill confidence as the athlete begins to solve the degrees-of-freedom problem associated with the assigned task. Further research is needed, and thus, providers are encouraged to use their clinical expertise to determine if and how to best use the immediate effects of plantar massage in their patients with CAI.

\section{CONCLUSIONS}

A single 5-minute session of plantar massage improved static postural control in those with CAI. A traditional plantar massage, a self-administered massage, and a sensory brush massage resulted in comparable static postural-control improvements. However, a single 5-minute session of plantar massage did not improve dynamic 
postural control in those with CAI. Finally, the results provide empirical evidence that the mechanism for the postural-control improvements is the stimulation of the plantar cutaneous receptors and not the stimulation of the underlying musculotendinous receptors.

\section{REFERENCES}

1. Hertel J. Functional anatomy, pathomechanics, and pathophysiology of lateral ankle instability. J Athl Train. 2002;37(4):364-375.

2. Hubbard-Turner T, Turner MJ. Physical activity levels in college students with chronic ankle instability. J Athl Train. 2015;50(7):742747.

3. Anandacoomarasamy A, Barnsley L. Long term outcomes of inversion ankle injuries. Br J Sports Med. 2005;39(3):e14.

4. Valderrabano V, Hintermann B, Horisberger M, Fung TS. Ligamentous posttraumatic ankle osteoarthritis. Am J Sports Med. 2006;34(4): $612-620$

5. Hertel J. Sensorimotor deficits with ankle sprains and chronic ankle instability. Clin Sports Med. 2008;27(3):353-370, vii.

6. Wikstrom EA, Hubbard-Turner T, McKeon PO. Understanding and treating lateral ankle sprains and their consequences: a constraintsbased approach. Sports Med. 2013;43(6):385-393.

7. Hoch MC, McKeon PO, Andreatta RD. Plantar vibrotactile detection deficits in adults with chronic ankle instability. Med Sci Sports Exerc. 2012;44(4):666-672.

8. Powell MR, Powden CJ, Houston MN, Hoch MC. Plantar cutaneous sensitivity and balance in individuals with and without chronic ankle instability. Clin J Sport Med. 2014;24(6):490-496.

9. Burcal CJ, Wikstrom EA. Plantar cutaneous sensitivity with and without cognitive loading in people with chronic ankle instability, copers, and uninjured controls. J Orthop Sports Phys Ther. 2016; 46(4):270-276

10. Wikstrom EA, Naik S, Lodha N, Cauraugh JH. Balance capabilities after lateral ankle trauma and intervention: a meta-analysis. Med Sci Sports Exerc. 2009;41(6):1287-1295.

11. McKeon PO, Hertel J. Systematic review of postural control and lateral ankle instability, part II: is balance training clinically effective? J Athl Train. 2008;43(3):305-315.

12. Docherty CL, Moore JH, Arnold BL. Effects of strength training on strength development and joint position sense in functionally unstable ankles. J Athl Train. 1998;33(4):310-314.

13. Feger MA, Herb CC, Fraser JJ, Glaviano N, Hertel J. Supervised rehabilitation versus home exercise in the treatment of acute ankle sprains: a systematic review. Clin Sports Med. 2015;34(2):329346.

14. LeClaire JE, Wikstrom EA. Massage for postural control in individuals with chronic ankle instability. Athl Train Sports Health Care. 2012;4(5):213-219.

15. Hoch MC, McKeon PO. Joint mobilization improves spatiotemporal postural control and range of motion in those with chronic ankle instability. J Orthop Res. 2011;29(3):326-332.

16. Ross SE, Arnold BL, Blackburn JT, Brown CN, Guskiewicz KM. Enhanced balance associated with coordination training with stochastic resonance stimulation in subjects with functional ankle instability: an experimental trial. J Neuroeng Rehabil. 2007;4:47.

17. Gribble PA, Delahunt E, Bleakley CM, et al. Selection criteria for patients with chronic ankle instability in controlled research: a position statement of the International Ankle Consortium. $J$ Athl Train. 2014;49(1):121-127.

18. Carcia CR, Martin RL, Drouin JM. Validity of the Foot and Ankle Ability Measure in athletes with chronic ankle instability. $J$ Athl Train. 2008;43(2):179-183.

19. Wier LT, Ayers GW, Jackson AS, Rossum AC, Poston WS, Foreyt JP. Determining the amount of physical activity needed for long-term weight control. Int J Obes Relat Metab Disord. 2001;25(5):613-621.

20. Ross SE, Linens SW, Wright CJ, Arnold BL. Balance assessments for predicting functional ankle instability and stable ankles. Gait Posture. 2011;34(4):539-542.

21. Ross SE, Guskiewicz KM, Gross MT, Yu B. Balance measures for discriminating between functionally unstable and stable ankles. Med Sci Sports Exerc. 2009;41(2):399-407.

22. Hertel J, Olmsted-Kramer LC, Challis JH. Time-to-boundary measures of postural control during single leg quiet standing. $J$ Appl Biomech. 2006;22(1):67-73.

23. Munro AG, Herrington LC. Between-session reliability of the star excursion balance test. Phys Ther Sport. 2010;11(4):128-132.

24. McKeon PO, Wikstrom EA. Sensory-targeted ankle rehabilitation strategies for chronic ankle instability. Med Sci Sports Exerc. 2016; 48(5):776-784.

25. Hoch MC, Andreatta RD, Mullineaux DR, et al. Two-week joint mobilization intervention improves self-reported function, range of motion, and dynamic balance in those with chronic ankle instability. J Orthop Res. 2012;30(11):1798-1804.

26. Cruz-Diaz D, Lomas Vega R, Osuna-Perez MC, Hita-Contreras F, Martinez-Amat A. Effects of joint mobilization on chronic ankle instability: a randomized controlled trial. Disabil Rehabil. 2015; 37(7):601-610.

27. Ross SE, Linens SW, Wright CJ, Arnold BL. Customized noisestimulation intensity for bipedal stability and unipedal balance deficits associated with functional ankle instability. $J$ Athl Train. 2013;48(4):463-470.

28. Hoch MC, McKeon PO. Integrating contemporary models of motor control and health in chronic ankle instability: a review of the literature. Athl Train Sports Health Care. 2010;2(2):82-88.

29. McKay GD, Goldie PA, Payne WR, Oakes BW. Ankle injuries in basketball: injury rate and risk factors. Br J Sports Med. 2001;35(2): 103-108.

30. Perry SD, McIlroy WE, Maki BE. The role of plantar cutaneous mechanoreceptors in the control of compensatory stepping reactions evoked by unpredictable, multi-directional perturbation. Brain Res. 2000;877(2):401-406.

31. Wikstrom EA, McKeon PO. The effects of 2 weeks of sensorytargeted ankle rehabilitation strategies on single limb center of pressure velocity in those with chronic ankle instability. Med Sci Sports Exerc. 2014;46(suppl 5):583.

32. McKeon PO, Wikstrom EA. The immediate effects of sensorytargeted ankle rehabilitation strategies on instrumented single limb balance in those with chronic ankle instability. Med Sci Sports Exerc. 2014;46(suppl 5):694.

33. Smith SS, Cheng T, Kerr GK. The effect of extended wake on postural control in young adults. Exp Brain Res. 2012;221(3):329335 .

34. Kwon YH, Choi YW, Nam SH, Lee MH. The influence of time of day on static and dynamic postural control in normal adults. $J$ Phys Ther Sci. 2014;26(3):409-412.

Address correspondence to Erik A. Wikstrom, PhD, ATC, FACSM, University of North Carolina at Chapel Hill, 311 Woollen Gym, CB\#8605, Chapel Hill, NC 27599. Address e-mail to wikstrom@unc.edu. 